\title{
The Long-Term Effects of Maternal Deprivation Depend on the Genetic Background
}

\author{
B.A. Ellenbroek, Ph.D., and A.R. Cools, Ph.D.
}

The neurodevelopmental hypothesis of schizophrenia has led to a series of new animal models in which the long term consequences of early manipulations are investigated. We have recently shown that a single 24-hr period of maternal deprivation (at postnatal day (pnd) 9) increases apomorphine susceptibility and decreases prepulse inhibition in Wistar rats, viz. phenomena also seen in schizophrenic patients. In the present paper, we investigated whether the effects of maternal deprivation were dependent on a specific genetic background, by using different rat strains (Fischer 344 and Lewis) that differ in the Hypothalamus-Pituitary-Adrenal axis and in dopaminergic sensitivity. The data show that in Wistar rats, basal startle amplitude was not affected by maternal deprivation, but prepulse inhibition was reduced, and apomorphine susceptibility enhanced. In Fischer 344 rats on the other hand, neither basal startle amplitude, nor prepulse inhibition were affected, but apomorphine susceptibility was reduced. In Lewis rats, maternal deprivation significantly reduced basal startle amplitude, but did not affect prepulse inhibition or apomorphine susceptibility. The differential response to maternal deprivation can best be explained by differences in baseline dopamine sensitivity between the rat strains. Since a reduced prepulse inhibition and an enhanced susceptibility to apomorphine is also seen in schizophrenic patients, the data indicate that maternally deprived Wistar rats may represent an interesting developmental model for (aspects of) schizophrenia. [Neuropsychopharmacology 23:99106, 2000] (C) 2000 American College of Neuropsychopharmacology. Published by Elsevier Science Inc. All rights reserved
KEY WORDS: Animal models; Apomorphine; Maternal deprivation; Prepulse inhibition; Rat strains; Schizophrenia

Prepulse inhibition refers to the reduction of the startle response to a brief low-intensity non-startling stimulus (Hoffman and Ison 1980) and is often considered to represent an operational measure of sensorimotor gating (Ellenbroek and Cools 1995a; Braff 1993). Studies have

From the Department of Psychoneuropharmacology, Nijmegen, The Netherlands.

Address correspondence to: Dr. B.A. Ellenbroek, Department of Psychoneuropharmacology, PO Box 9101, 6500 HB Nijmegen, The Netherlands.

Received March 19, 1999; revised June 23, 1999; accepted January 4, 2000 . shown that prepulse inhibition is reduced in schizophrenic patients (Braff et al. 1978). Since prepulse inhibition can also be measured in animals with virtually identical methods, it is often used as an animal model for (aspects of) schizophrenia (Ellenbroek and Cools 1990).

The most important question is how to simulate the schizophrenic-like deficit in prepulse inhibition. Many groups have initially focussed on pharmacological alterations in prepulse inhibition. For instance, dopamine agonists (Mansbach et al. 1988), non competitive NMDA antagonists (Mansbach and Geyer 1989), and serotonine agonists (Sipes and Geyer 1995) have been found to disrupt prepulse inhibition. The recent hypothesis that schizophrenia most likely results from 
disturbances in the normal development (Pilowsky et al. 1993) has led to a whole new category of animal models, in which the long term consequences of manipulations early in development, rather than the acute effects of drugs are studied (Ellenbroek and Cools 1998a). These manipulations include neonatal hippocampal lesions (Lipska et al. 1995) and isolation rearing (Geyer et al. 1993).

Previous research on both the pharmacological and the developmental models has clearly shown that the effects strongly depend on the strain of rats used. Thus, Wistar rats are more sensitive to the disrupting effects of apomorphine than Sprague Dawley rats (Kinney et al. 1999), which in turn are more sensitive than Lewis rats (Varty and Geyer 1998). Likewise, isolation rearing disrupts prepulse inhibition in Sprague Dawley and Fischer 344 rats, has no effects in Lewis and Wistar rats, and seems to potentiate prepulse inhibition in Fawn Hooded rats (Hall et al. 1997; Varty and Geyer 1998). It is at present unclear why these strains differ in their response to these manipulations, especially since they generally do not show differences in prepulse inhibition per se. It has been suggested that differences in neuroendocrine and/or neurochemical (more specifically dopaminergic) state may be responsible for the differential response to drugs and early manipulation (Varty and Geyer 1998; Kinney et al. 1999).

We have recently shown that a single period of $24 \mathrm{hr}$ of maternal deprivation in Wistar rats leads to a disruption of prepulse inhibition which can be temporarily reversed by antipsychotic drugs such as haloperidol and quetiapine, and which only occur after puberty (Ellenbroek et al. 1998b).

The aim of the present study was to investigate whether other strains showed similar responses to early maternal deprivation. More specifically, we investigated the long term consequences of maternal deprivation in Fischer 344 and Lewis rats and compared these with the effects in Wistar rats. Basal startle amplitude, prepulse inhibition and susceptibility to apomorphine were used as dependent variables. Fisher 344 and Lewis rats were chosen for three reasons. First of all, the effects of pharmacological and early environmental manipulation on prepulse inhibition has usually been studied in these two rat strains, which makes comparison between these models easier. Secondly, these rats differ in hypothalamic-pituitary-adrenal axis reactivity. Fischer 344 rats show a much stronger corticosterone and ACTH response to a stressful stimulus than Lewis rats, whereas Wistar rats show an intermediate response (Sternberg et al. 1992).

Since maternal deprivation leads to alterations in this axis (Levine 1994), potential differences in response to maternal deprivation may be explained by differences in baseline HPA-axis reactivity. Thirdly, Fischer 344 and Lewis rats differ in the susceptibility to dopamimetics such as cocaine (Camp et al. 1994). In a recent study, we could show that Wistar rats showed a much stronger stereotyped gnawing response than Fischer 344 rats, with Lewis rats showing an intermediate response (Ellenbroek and Cools 1996). Since early maternal deprivation increases the gnawing response to apomorphine (Rots et al. 1996), potential differences in response to maternal deprivation between the three strains may be explained by differences in baseline dopaminergic susceptibility.

\section{MATERIALS AND METHODS}

Male and (primiparous) female Wistar, Lewis, and Fischer 344 rats were obtained from and housed in the Central Animal laboratory of the University of Nijmegen in standard temperature controlled rooms, on a standard 12-hr light/dark cycle (light on at 0700 hr). The rats had free access to food and water, and were initially housed in pairs of one male and one female of each strain in a Macrolon ${ }^{\circledR}$ cage $(26 \times 42 \times 15$ $\mathrm{cm})$. When the females were clearly pregnant, the males were removed from the cage and the females were checked twice daily (0800 and $1700 \mathrm{hr}$ ) for delivery.

The day of delivery was designated postnatal day (pnd) 0. Maternal deprivation took place at pnd 9. The mothers were removed from the cage around $1000 \mathrm{hr}$ and placed in a single cage. The pups were weighed and left in the homecage at room temperature. No food was available during the deprivation period. In control litters, mothers were removed at pnd 9, pups were weighed and mothers returned immediately (within 3 minutes). At pnd 10 (24 hr later), the maternally deprived pups were weighed again and the mothers placed back. In the control litter the mothers were again removed, pups weighed and mothers placed back. The litters were left undisturbed except for cleaning of the cage once a week until weaning (pnd 21), after which the males were housed in groups of two or three per cage. Rats were individually housed at pnd 68 , and testing took place at pnd 69. Experiments were done on male rats only, and each rat was used only once.

In the prepulse inhibition paradigm, four standard startle chamber of San Diego Instruments were used. The startle chamber consisted of a Plexiglas tube (diameter $8.2 \mathrm{~cm}$, length $25 \mathrm{~cm}$ ), placed in a sound attenuated chamber, in which the rats were individually placed. The tube was mounted on a plastic frame, under which a piezoelectric accelerometer was mounted, which recorded and transduced the motion of the tube. After the rats were placed into the chamber they were allowed to habituate for a period of five minutes during which a 70 $\mathrm{dB}[\mathrm{A}]$ background white noise was present. After this period, the rats received 10 startle trials, 10 no-stimulus trials, and 30 prepulse inhibition trials. The intertrial in- 
terval was between 10 and 20 seconds and the total session lasted about 17 minutes.

The startle trial consisted of a single $120 \mathrm{~dB}[\mathrm{~A}]$ white noise burst lasting $20 \mathrm{~ms}$. The prepulse inhibition trials consisted of a prepulse (20 ms burst of white noise with intensities of 73, 75, or $80 \mathrm{db}[\mathrm{A}]$ ) followed, $100 \mathrm{~ms}$ later, by a startle stimulus (120 dB[A], $20 \mathrm{~ms}$ white noise). Each of the three prepulse trials $(73,75$, and $80 \mathrm{~dB}[\mathrm{~A}])$ were presented 10 times. During the no-stimulus trial, no stimulus is presented but the movement of the rat is scored. This represents a control trial for detecting differences in overall activity. Since no such differences were detected, the results are not shown. The 50 different trials were presented pseudo-randomly, ensuring that each trial was presented 10 times and that no two consecutive trials were identical. The resulting movement of the rat in the startle chamber was measured during $100 \mathrm{~ms}$ after startle stimulus onset (sampling frequency $1 \mathrm{kHz}$ ), rectified, amplified, and fed into a computer which calculated the maximal response over the $100 \mathrm{~ms}$ period. Basal startle amplitude was determined as the mean amplitude of the 10 startle trials. Prepulse inhibition was calculated according to the formula $100-100 \% *(\mathrm{PPx} / \mathrm{P} 120)$, in which PPx is the mean of the 10 prepulse inhibition trials (PP73, PP75, or PP80) and P120 is the basal startle amplitude.

Differences in basal startle amplitude were analysed by a two-way analysis of variance (ANOVA) with factors early manipulation (deprived vs. nondeprived) and strain (Fischer 344, Lewis and Wistar). The overall effect on prepulse inhibition was determined by an ANOVA with repeated measures for the different prepulse intensities and early manipulation and strain as between factors. In case of a significant effect, post hoc Duncan tests were performed to evaluate the statistical differences between the groups for each prepulse intensity.

In the apomorphine susceptibility test, rats were subcutaneously injected with $1.5 \mathrm{mg} / \mathrm{kg}$ apomorphine- $\mathrm{HCl}$ (Brocades, ACF, Maarssen, The Netherlands) or with saline and placed in a gnawing box (modified after Ljungberg and Ungerstedt 1978). The gnawing box consisted of a Perspex hole board $(69 \times 69 \mathrm{~cm})$ with a central cubicle $(25 \times 25 \mathrm{~cm})$. The box contained 32 holes, each of which was surrounded by five concentric ridges. A microphone placed underneath the central cubicle allowed registration of sounds. Through this microphone and a large number of infrared beams, a number of behavioural activities were automatically recorded, like locomotor activity, frequency and duration of hole dipping, etc. (Cools et al. 1990). For the present experiments, only the stereotyped gnawing response and the motor activity were determined. The gnawing on the ridges surrounding the holes produced a characteristic sound which was detected by the microphone, fed into the computer and scored as a gnawing count. Motor activity was recorded by calculating the total number of interrupted infrared beams over 45 minutes. Differences in gnawing response and motor activity were determined with a three way ANOVA: factor drug (apomorphine vs. saline) and factors early manipulation and strain.

\section{RESULTS}

The effects of maternal deprivation on basal startle amplitude and prepulse inhibition are depicted in Figure 1. The ANOVA on basal startle amplitude showed significant effects of early manipulation $(\mathrm{F}(1,71)=7.7, p<$ $.007)$ and of strain $(\mathrm{F}(2,71)=14.9, p<.001)$. The interaction was also significant $(\mathrm{F}(1,71)=3.1, p<.05)$. Post hoc tests showed that maternal deprivation did not affect basal startle amplitude in Fischer 344 and Wistar rats, but significantly reduced it in Lewis rats. Moreover, the analysis showed that Lewis rats had a significantly larger basal startle amplitude than Wistar or Fischer 344.

The effects of maternal deprivation on prepulse inhibition were also strain dependent, as can be seen in Figure 1B. The ANOVA showed a significant effect of prepulse intensity $((\mathrm{F}(2,142)=94.6, p<.007)$, of early manipulation $(\mathrm{F}(1,71)=4.2, p<.05)$, but not of strain $(\mathrm{F}(2,71)=2.5, p=.09)$. The interaction between early manipulation and strain, however, did reach significance $(F(2,71)=4.2 p<.02)$. Post hoc tests showed that maternal deprivation significantly reduced prepulse inhibition in Wistar rats, but had no effect on Fischer 344 or Lewis rats.

The effects of maternal deprivation on apomorphine susceptibility are shown in Figure 2.. When looking at activity (Figures 2A, B, and C), there was no significant drug effect $(\mathrm{F}(1,97)=2.5, p=.11)$, but a significant effect of strain $(\mathrm{F}(2,97)=6.6, p<.002)$, and a significant drug $\times$ strain interaction $(\mathrm{F}(2,97)=4.2, p<.02)$ as well as a significant three way interaction drug $\times$ early manipulation $\times$ strain $(\mathrm{F}(2,97)=4.4, p<.02)$. Post hoc analysis showed that apomorphine reduced the activity in maternally deprived Wistar rats (but not in control Wistar rats) whereas the reverse was true in Fischer 344: a reduction in control rats, but no effect in maternally deprived rats.

When looking at the effects on gnawing (Figures 2D, $\mathrm{E}$, and $\mathrm{F})$, there was a significant effect of drug $(\mathrm{F}(1,97)=$ $4.3, p<.05)$ and strain $(\mathrm{F}(2,97)=3.7, p<.03)$. Moreover, the two way drug $\times$ strain interaction was significant $(\mathrm{F}(2,97)=3.4, p<.04)$, as well as the three-way interaction drug $\times$ early manipulation $\times$ strain $(\mathrm{F}(2,97)=$ $4.1, p<.02)$. Post hoc analysis showed that apomorphine increased gnawing in maternally deprived Wistar rats, but not in control Wistar rats (Figure 2D). Neither control nor maternally deprived Fischer 344 rats showed an increased gnawing response after apomorphine (Figure 2E), whereas both control and mater- 

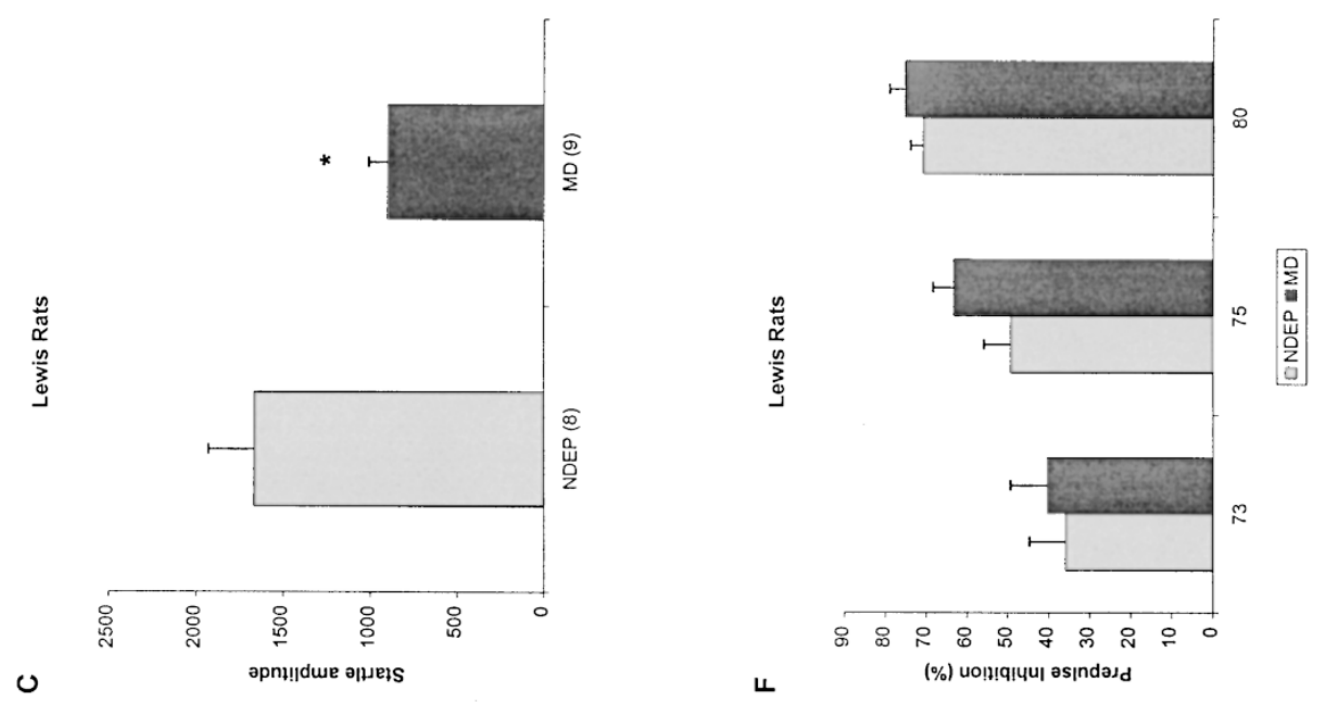

促
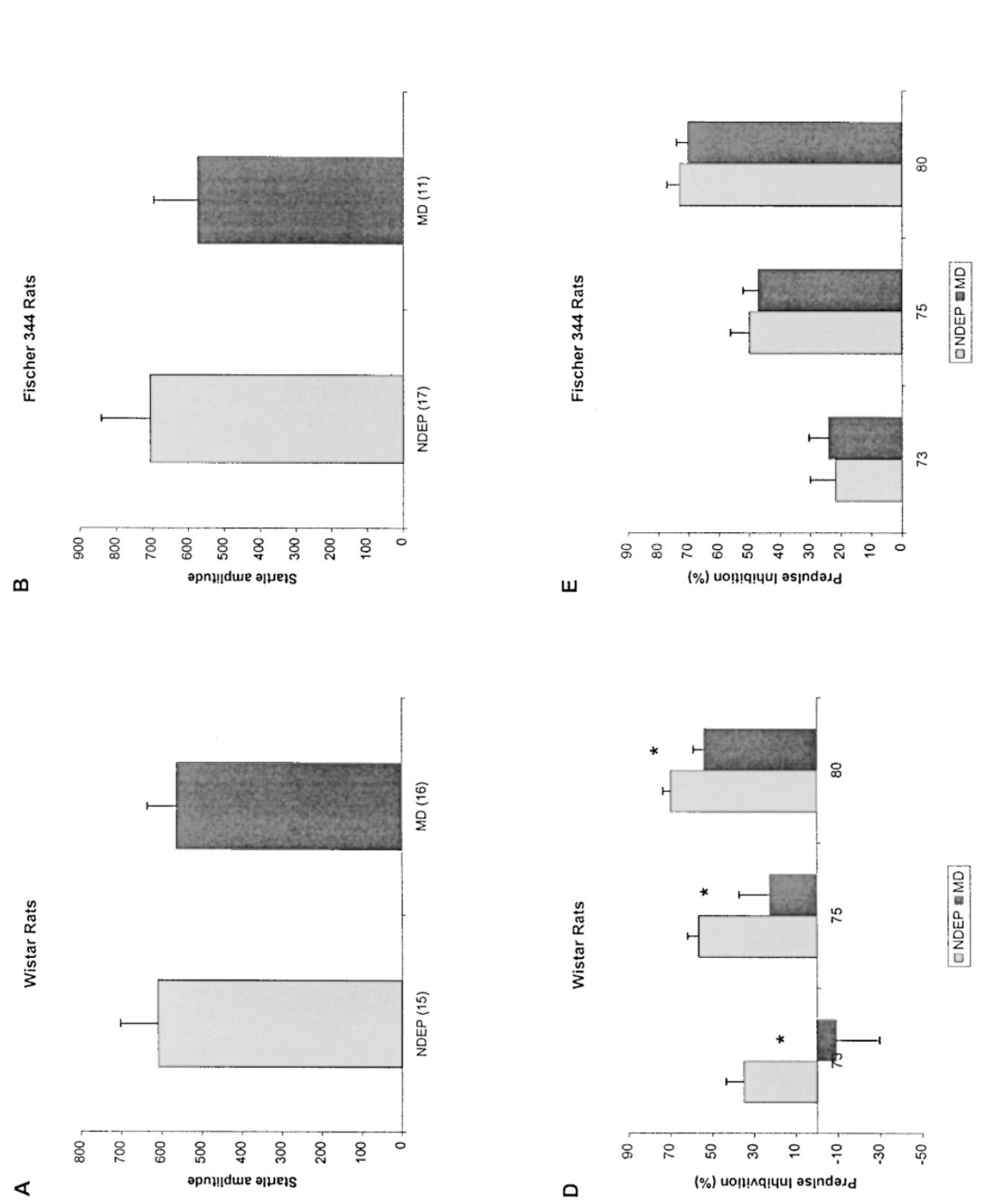

山

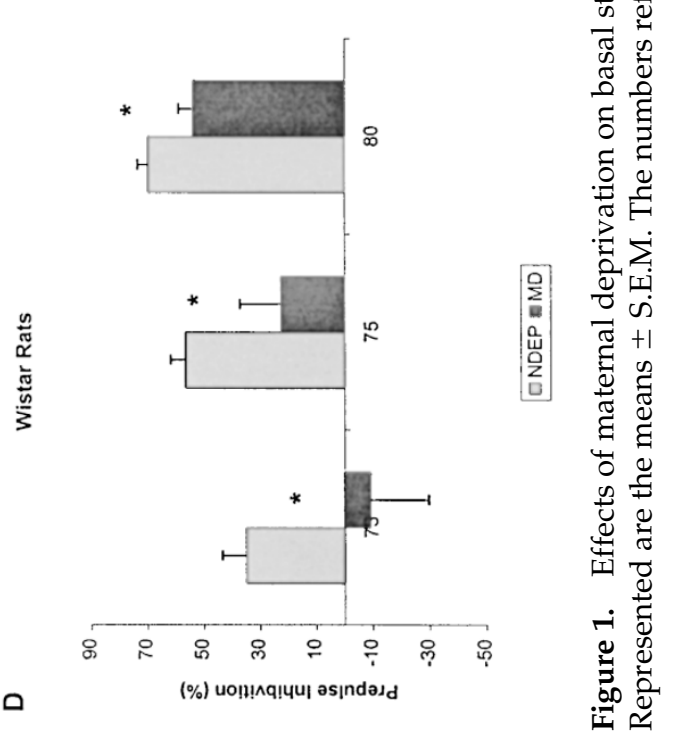



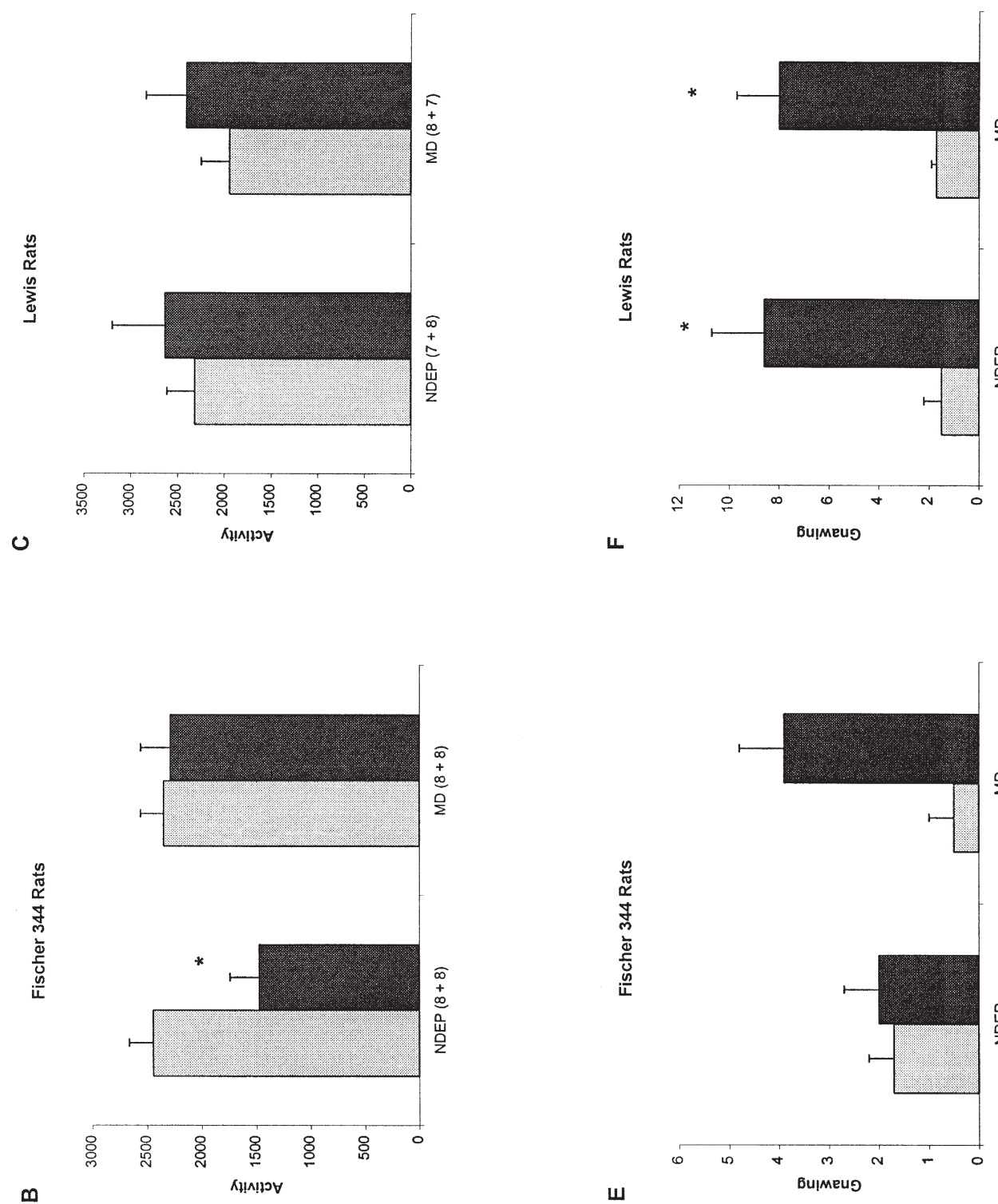

เ
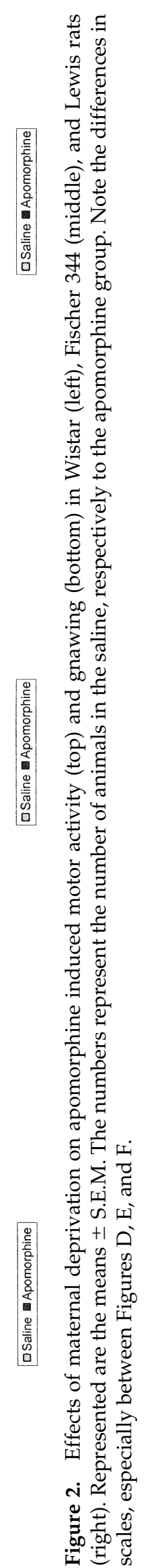
nally deprived Lewis rats showed an increased gnawing response after apomorphine.

\section{DISCUSSION}

The results of the present experiments show that the long-term effects of maternal deprivation depend on the strain of rat, as well as on the parameter studied. This is most clearly illustrated for basal startle amplitude and prepulse inhibition. Maternal deprivation reduced basal startle amplitude only in Lewis rats, and reduced prepulse inhibition only in Wistar rats. With respect to the effects of maternal deprivation on apomorphine susceptibility, the situation is more complex. In Wistar rats, maternal deprivation led to an enhanced apomorphine induced gnawing, and a decrease in apomorphine induced general activity. Under normal circumstances, low doses of apomorphine increase locomotor activity, whereas higher doses reduce locomotor activity and increase gnawing. Thus, these data can be interpreted as an increased sensitivity of maternally deprived Wistar rats to apomorphine. In Fischer 344 rats, apomorphine reduced motor activity in control, but not in deprived rats. Following the reasoning outlined above, this may be interpreted as a reduced susceptibility to apomorphine after maternal deprivation.

The data show that there were significant differences between the three strains with respect to basal startle amplitude. Figure 1A showed that control Lewis rats had a more than two-fold higher basal amplitude (1666) than both Fischer 344 (704) and Wistar rats (612). This is in agreement with the data obtained by others (Glowa et al. 1992). It is at present unclear which mechanisms govern this increase in startle amplitude. Although it has been suggested that startle may be related to anxiety, this seems an unlikely explanation, since Lewis and Fischer 344 rats do not differ in other anxiety related measures, such as behaviour in the elevated plus maze or black and white box (Chaouloff et al. 1995).

Glowa and colleagues (1992) have suggested that basal startle amplitude may be negatively correlated to HPA axis activation. Although this would explain the higher baseline startle response in Lewis rats, it would also imply that maternal deprivation in Wistar rats should lead to changes in basal startle amplitude, since this procedure is known to alter the HPA axis (Rots et al. 1996). The present data (Figure 1) and previous results (Ellenbroek et al. 1998b) clearly show that this is not the case. In contrast to the strain differences observed in startle amplitude, no differences were found between the strains in prepulse inhibition. Although differences in prepulse inhibition have been reported between Fawn Hooded and Wistar rats (Hall et al. 1997), most studies have found no big differences between strains. In particular, no differences were found between Fischer 344, Lewis, and Sprague Dawley rats (Varty and Geyer 1998), nor between Sprague Dawley and Wistar rats (Swerdlow et al. 1998; Kinney et al. 1999).

The finding that prepulse inhibition is reduced in maternally deprived Wistar rats is in agreement with our previous results (Ellenbroek et al. 1998b). Varty and Geyer (1998) recently showed that isolation rearing (i.e., rats housed in separate cages from weaning) disrupted prepulse inhibition in Sprague Dawley and Fischer 344 rats, but not in Lewis rats. Moreover, they found that low doses of apomorphine which disrupt prepulse inhibition in Sprague Dawley and Fischer 344 rats did not affect prepulse inhibition in Lewis. Likewise, Lipska and Weinberger (1995) found that Lewis rats did not show an increased response to amphetamine after early hippocampal lesions. Our data seem to be in general agreement with these finding since Lewis rats were also resistant to maternal deprivation (at least with respect to prepulse inhibition and apomorphine susceptibility). Overall, this suggests that Lewis rats are relatively resistant in these models for the neurodevelopmental hypothesis of schizophrenia (Ellenbroek and Cools 1998a). However, both Varty and Geyer (1998) and Lipska and Weinberger (1995) found that Fischer 344 rats were quite sensitive to isolation rearing, respectively to neonatal hippocampal lesions. Our data show that, at least with respect to maternal deprivation, Fischer 344 rats are much less sensitive than Wistar, which is likely to result from the difference in the type of manipulation.

There are also clear strain differences in apomorphine sensitivity. In Wistar rats, apomorphine increased stereotyped gnawing; in Fischer 344 rats, apomorphine reduced general motor activity; whereas in Lewis rats there was a slight but significant increase in stereotyped gnawing. These data are in general agreement with previous reports, showing that apomorphine is more effective in inducing gnawing in Wistar rats than in either Lewis or Fischer 344 rats (Ellenbroek and Cools 1996). The effects of maternal deprivation on apomorphine susceptibility are more complicated. In Wistar rats, the apomorphine induced gnawing is significantly enhanced after maternal deprivation, which is in agreement with previous results (Rots et al. 1996;Ellenbroek and Cools 1995b). In Lewis rats, maternal deprivation had no influence on apomorphine susceptibility. In Fischer 344, however, maternal deprivation led to a reduction in apomorphine susceptibility. While apomorphine significantly reduced motor activity in non deprived Fischer 344 rats, it had no effect of deprived rats.

Overall, the results show that, depending on the rat strain, maternal deprivation led to different long term effects. Since an increase in apomorphine susceptibility (Muller et al. 1998) and a decreased in prepulse inhibition (with no difference in basal startle amplitude) has 
been reported for schizophrenic patients (Braff et al. 1978), the present data suggest that the maternally deprived Wistar rat may represent an interesting model, especially since these rats show other abnormalities also occurring in schizophrenic patients (Ellenbroek and Cools 1995b).

In the introduction, three possibilities were mentioned for explaining potential differences between the three strains: 1) difference in baseline prepulse inhibition; 2) difference in baseline HPA-axis response; and 3) difference in baseline apomorphine response. The lack of difference between the three lines in baseline prepulse inhibition (see Figure 1), rules out the first possibility. If possibility 2 was correct, one would expect Fischer 344 rats to show the largest (or the smallest) deficit, followed by Wistar and Lewis rats, given the much higher stress sensitivity of Fischer 344 rats response (Sternberg et al. 1992). If possibility 3 was correct, the Wistar should show the strongest (or the weakest) deficit, followed by Lewis and Fischer 344 rats, given the much higher sensitivity of Wistar rats for apomorphine (see Figure 2). When focussing on prepulse inhibition, Wistar rats clearly showed the strongest deficit, with Fischer 344 and Lewis showing no deficit. Likewise, when focussing on apomorphine sensitivity, Wistar rats showed a strong increase, Lewis no effect, and Fischer 344 rats showed a decrease. These data suggest that the differences in sensitivity of the lines to maternal deprivation is related to differences in baseline dopaminergic sensitivity. In this respect, it is interesting to note that maternal deprivation in Wistar rats led to an increase in mRNA for tyrosine hydroxylase within the substantia nigra pars compacta (Rots et al. 1996). It would be interesting to see whether maternal deprivation affects tyrosine hydroxylase in Fischer 344 and Lewis as well.

In summary, the present data show that early maternal deprivation induced schizophrenia-like abnormalities in adult Wistar rats, but not in Fischer 344 or Lewis rats. These data provide strong support for the general hypothesis that genetic and environmental factors may contribute to the overall development of the brain and brain function, and suggest that maternal deprivation may represent a novel and very interesting tool for studying the interaction between genetic and early stressful life events.

\section{REFERENCES}

Braff D (1993): Information processing and attention dysfunctions in schizophrenia. Schizophr Bull 19:233-260

Braff D, Stone C, Callaway E, Geyer MA, Glick ID, Bali L (1978): Prestimulus effects of human startle reflex in normals and schizophrenics. Psychophysiology 15:339-343

Camp DM, Browman KE, Robinson TE (1994): The effects of methamphetamine and cocaine on motor behavior and extracellular dopamine in the ventral striatum of Lewis versus Fischer 344 rats. Brain Res 668:180-193

Chaouloff F, Kulikov A, Sarrieau A, Castanon N, Mormede P (1995): Male Fischer 344 and Lewis rats display differences in locomotor reactivity, but not in anxiety-related behaviours: Relationship with the hippocampal serotonergic system. Brain Res 693:169-178

Cools AR, Brachten R, Heeren D, Willemen A, Ellenbroek B (1990): Search after neurobiological profile of individualspecific features of Wistar rats. Brain Res Bull 24:49-69

Ellenbroek BA, Cools AR (1990): Animal models with construct validity for schizophrenia. Behav Pharmacol 1:469-490

Ellenbroek BA, Cools AR (1995a): Animal models of psychotic disturbances. In den Boer JA, Westenberg HGM, van Praag HM (eds), Advances in the Neurobiology of Schizophrenia. Chichester UK, John Wiley \& Sons Ltd, pp 89-109

Ellenbroek BA, Cools AR (1995b): Maternal separation reduces latent inhibition in the conditioned taste aversion paradigm. Neurosci Res Comm 17:27-33

Ellenbroek BA, Cools AR (1996): Dopamine susceptibility and information processing. In Beninger RJ, Palamo T, Archer T (eds), Dopamine Disease States. Strategies for Studying Brain Disorders, Vol. 3. Madrid, Spain, Editorial Cym, pp 447-462

Ellenbroek BA, Cools AR (1998a): The neurodevelopmental hypothesis of schizophrenia: Clinical evidence and animal models. Neurosci Res Comm 22:127-136

Ellenbroek BA, van-den KPTJM, Cools AR (1998b): The effects of an early stressful life event on sensorimotor gating in adult rats. Schizophr Res 30:251-260

Geyer MA, Wilkinson LS, Humby T, Robbins TW (1993): Isolation rearing of rats produces a deficit in prepulse inhibition of acoustic startle similar to that in schizophrenia. Biol Psychiatry 34:361-372

Glowa JR, Geyer MA, Gold PW, Sternberg EM (1992): Differential startle amplitude and corticosterone response in rats. Neuroendocrinology 56:719-723

Hall FS, Huang S, Fong G (1997): Effects of isolation-rearing on acoustic startle and pre-pulse inhibition in Wistar and fawn hooded rats. Ann NY Acad Sci 821:542-544

Hoffman HS, Ison JR (1980): Reflex modification in the domain of startle. I. Some empirical findings and their implications for how the nervous system processes sensory input. Psychol Rev 87:175-189

Kinney GG, Wilkinson LO, Saywell KL, Tricklebank MD (1999): Rat strain differences in the ability to disrupt sensorimotor gating are limited to the dopaminergic system, specific to prepulse inhibition, and unrelated to changes in startle amplitude or nucleus accumbens dopamine receptor sensitivity. J Neurosci 19:5644-5653

Levine S (1994): The ontogeny of the hypothalamic-pituitary-adrenal axis. The influence of maternal factors. Ann NY Acad Sci 746:275-288

Lipska BK, Chrapusta SJ, Egan MF, Weinberger DR (1995): Neonatal excitotoxic ventral hippocampal damage alters dopamine response to mild repeated stress and to chronic haloperidol. Synapse 20:125-130 
Lipska BK, Weinberger DR (1995): Genetic variation in vulnerability to the behavioral effects of neonatal hippocampal damage in rats. Proc Natl Acad Sci 92:S906-S910

Ljungberg T, Ungerstedt U (1978): A new method for simultaneous registration of 8 behavioral parameters related to monoamine neurotransmission. Pharmacol Biochem Behav 8:483-489

Mansbach RS, Geyer MA (1989): Effects of phencyclidine and phencyclidine biologs on sensorimotor gating in the rat. Neuropsychopharmacology 2:299-308

Mansbach RS, Geyer MA, Braff DL (1988): Dopaminergic stimulation disrupts sensorimotor gating in the rat. Psychopharmacology 94:507-514

Muller SF, Modell S, Ackenheil M, Brachner A, Kurtz G (1998): Elevated response of growth hormone to graded doses of apomorphine in schizophrenic patients. J Psychiatry Res 32:265-271

Pilowsky LS, Kerwin RW, Murray RM (1993): Schizophrenia: A neurodevelopmental perspective. Neuropsychopharmacology 9:83-91
Rots NY, de JJ, Workel JO, Levine S, Cools AR, de KE (1996): Neonatal maternally deprived rats have as adults elevated basal pituitary-adrenal activity and enhanced susceptibility to apomorphine. J Neuroendocrinol 8:501-506

Sipes TA, Geyer MA (1995): 8-OH-DPAT disruption of prepulse inhibition in rats: Reversal with $(+)$ WAY 100,135 and localization of site of action. Psychopharmacology 117:41-48

Sternberg EM, Glowa JR, Smith MA, Calogero AE, Listwak SJ, Aksentijevich S, Chrousos GP, Wilder RL, Gold PW (1992): Corticotropin releasing hormone related behavioral and neuroendocrine responses to stress in Lewis and Fischer rats. Brain Res 570:54-60

Swerdlow NR, Varty GB, Geyer MA (1998): Discrepant findings of clozapine effects on prepulse inhibition of startle: is it the route or the rat? Neuropsychopharmacology 18:50-56

Varty GB, Geyer MA (1998): Effects of isolation rearing on startle reactivity, habituation, and prepulse inhibition in male Lewis, Sprague-Dawley, and Fischer F344 rats. Behav Neurosci 112:1450-1457 